\title{
Study on Acquisition System of Diversification Heartbeat Information based on Body Sensor Network
}

\author{
Junrong Bao ${ }^{1}$, Wenfeng $\mathrm{Li}^{2}$, Bizhen $\mathrm{Lian}^{3}$,Jian $\mathrm{Li}^{4}$,Yanhong $\mathrm{Ge}^{2}$, Jiagen $\mathrm{Jin}^{2}$ \\ ${ }^{1}$ School of Computer Science and Technology, Wuhan University of Technology, Wuhan, China \\ ${ }^{2}$ School of Logistics Engineering, Wuhan University of Technology, Wuhan, China \\ ${ }^{3}$ Beijing Sport University, Beijing, China \\ ${ }^{4}$ Pilot Physical Examination Center of Lanzhou, Lanzhou, China \\ bjr@whut.edu.cn, liwf@whut.edu.cn, lianbizhen@yahoo.com.cn, 36773308@qq.com,gyh1898@163.com, jinjiagen@126.com
}

\begin{abstract}
Heartbeat information and motion parameters have a close relationship with human's health and emotion. The feasibility of acquisition heartbeat information associated with emotional states based on body sensor network is a hot research topic. In this paper, we investigate four kinds of method to collect heartbeat information, which contain complementary information of different biological information dimension. This study truly displays the concept of biological information system theory, which can collect heartbeat information with all of a human body's biological system information. The information collected from several collecting methods will be validated from various angles for each dimension.

Index Terms - Heartbeat Information; Sensor; Data Fusion; Biological Information Systemic; Body Sensor Networks;
\end{abstract}

\section{Introduction}

Remote home monitoring of symptoms and physiological variables may allow early detection and treatment of many physical and mental illness, there are growing evidences to support that physical illness and mental illness are very closely linked[1].The biological signals based on body sensor networks may carry specific characteristics that reflect basic dynamics of the human body[2].Therefore, acquiring a full range of information of the person by body sensor network is a hot topic $[3,4]$, reviewing the ancient and modern literature of traditional Chinese medicine and western medicine, The human heartbeat information is a well-studied topic, heartbeat signals carry specific signatures that are related to human physiologic mechanisms and psychological mechanisms[5], such as tachycardia is a resting heart rate more than 100 beats per minute. It can be led emotional conditions such as anxiety or stress, human motion, and so on. Athletes are interested in monitoring their heartbeat information to gain maximum efficiency from their training. Heartbeat information include emotion factor[6, 7], movement factor, and about 174 pathology Irregular heartbeats[8], and so on. The heartbeat information analysis has made positive contributions to the timely detection and better management of physical illness and mental illness in home or clinical situations[9].

Heartbeat information monitors allow measurements to be taken continuously and can be used during exercise when manual measurement would be difficult. Traditional, the objective heartbeat information can use a few means to achieve. such as the measurement of heart rate usually can be measured by checking the pulse or listening to the heartbeat with a stethoscope with doctor. At present, The first, heartbeat information might obtain through probing the bioelectrical of drive heartbeat, and ECG is precise method of determining heartbeat information with this theory; the second, it can be obtained by pulse wave according to Chinese medicine; the third, it can also get by blood oxygen volume. The fourth, photoplethysmogram(PPG), This optical technique measures heart rate by monitoring the subtle changes in skin color as the capillaries in the tissue expand and contract with each heartbeat[10, 11].

The characterizations of heartbeat information have heart rate, waveform shape features, the electrical activity of the heartbeat. Heart rate, the speed of the heartbeat, can vary as the body's need to absorb oxygen and excrete carbon dioxide changes, such as during physical exercise, sleep or illness.

However, as science and technology was the rapid development, many new methods is been emergence. Professor William T. Freeman, Massachusetts Institute of Technology, they are able to visualize the flow of blood as it fills the face using Eulerian Video Magnification framework for visualizing the human pulse[12], Daniel Lakens demonstrates the feasibility of measuring heart rate differences associated with emotional states such as anger and happiness with a Smartphone [6], in this paper we practice a new method to obtain heart rate by pressure cushion recording of the body vibrations with body sensor network. In the future, sensing data of clothes on our body and around the body of the sensor transmit our physiologic and psychological information to specific doctor in real time.

\section{II . Obtaining heartbeat information with pressure cushion}

\section{A. raw data of collecting system}

In this paper, the architecture of acquisition heartbeat information system is proposed, depicted in

Fig. 1 , they are basically composed of three modules: sensing, processing and transmitting. Sensing module is composed of four pressure sensor; processing module is used AVR single-

\footnotetext{
* This research is partly sponsored by the Ministry of Science and Technology of China (Grant Number: 2012BAJ05B07), International cooperation projects of Hubei province (2011BFA012)
} 
chip microprocessor; and transmitting module is collected raw pressure data to a central coordinator with Wifi[13] , depicted in Fig.2 and Fig.3.

The actual acquisition system has established that pressure data can be collected from a group of FSR sensors. A family of curves can be obtained which is a nominal force vs. output voltage curve, then the values of accuracy force of the system can be obtained. Ultimately the pressure distribution variability of real time was obtained from the system.

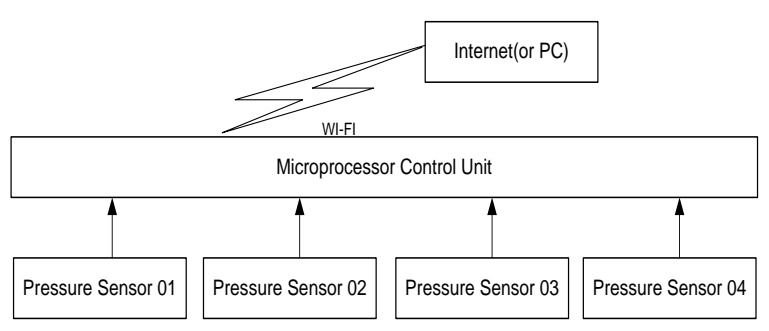

Fig. 1 Collecting system layout

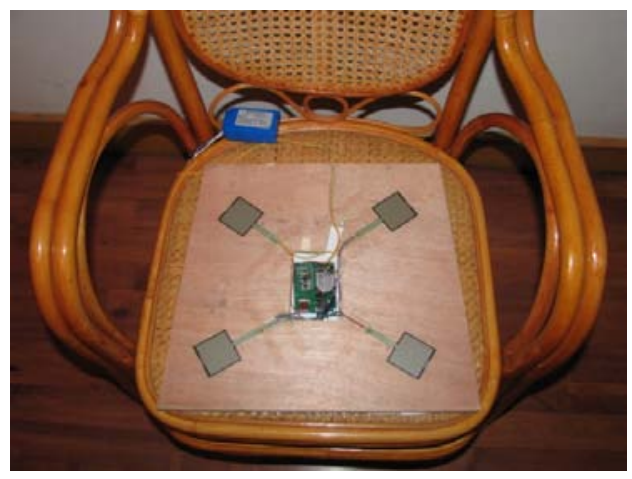

Fig. 2 Actual system layout

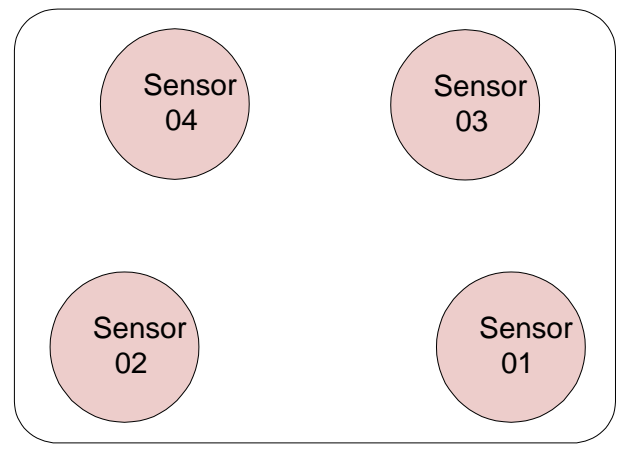

Fig. 3 Pressure sensors layout

FSR (Force Sensitive Resistor) sensor is majorly sensing unit, it will vary its resistance depending on how much pressure is being applied to the sensing area. The force is the greater, and the resistance is the lower. show Fig. 4, We assume the following value interval in this system, the maximum resistance of the pressure sensor defines $5 \mathrm{k} \Omega$, the pressure value corresponds to $100 \mathrm{~g}$, and the minimum resistance of the pressure sensor defines $200 \Omega$, the pressure value corresponds to $10 \mathrm{~kg}$.It make full use of single sensor elements as well as integrated arrays of sensors to obtain real timely pressure distribution variability due to the beating of the heart.

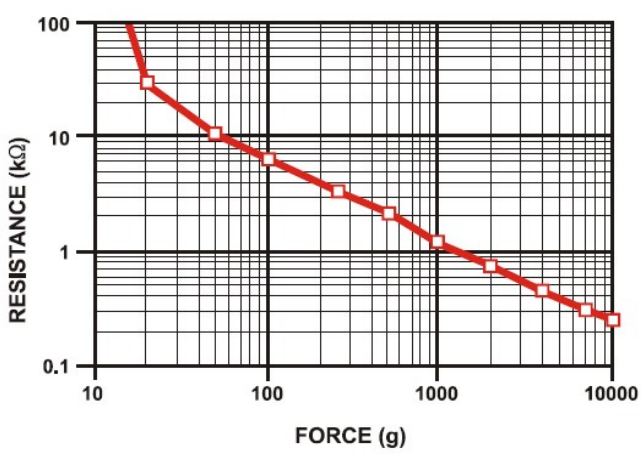

Fig. 4 Force vs. Output resistance curve

\section{B. Dealing Of Collected Data}

The analogue smoothing and filter design is essential for proper pulse readout. There are still some things to improve on this part. A real big improvement can be achieved by using active filters of a higher order to obtain a much sharper filter response, really leaving only the frequencies between about $1 \mathrm{~Hz}$ and 20Hz.show Fig. 5 ,Fig. 6 .

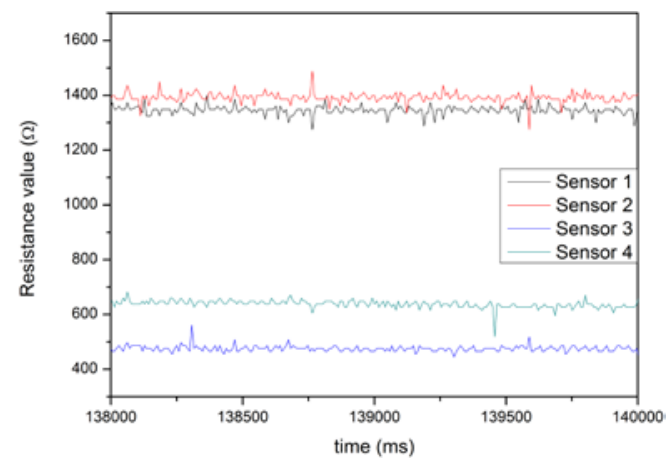

Fig. 5 time series of pressure sensor

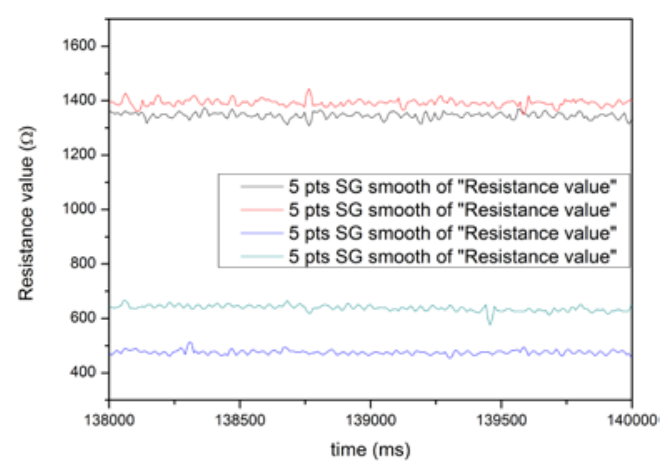

Fig. 6 time series with smoothing of pressure sensor

It is explored the relationship between the pressure sensors variability because of heartbeat and heart rate; based on data 
fusion establish the evaluation model of heart rate. A heart rate extracting method and apparatus which are capable of generating an accurate heart rate output which the heartbeat input signal greatly pulsates. Input signal where the low-frequency noise component is superimposed on a pulse waveform which is the signal component so as to obtain an amplified signal. signal is inputted to a low-pass filter so as to output only the amplified flow frequency component The instances verify that the model can accurately predict the relations between pressure distribution variability and heart rate.

\section{Obtaining heartbeat information with piezoelectric pulse sensor}

The real output waveform of the pulse wave, which varies from person to person, weather differences and environmental differences. non-invasive pulse signals collected by piezoelectric sensors, it can be measured at any point on the body where the artery's pulsation is transmitted to the surface by pressuring it, however, according to contemporary sphygmology in traditional Chinese medicine ,Heartbeat information is best measured by the wrist of the human, show Fig. 7 .

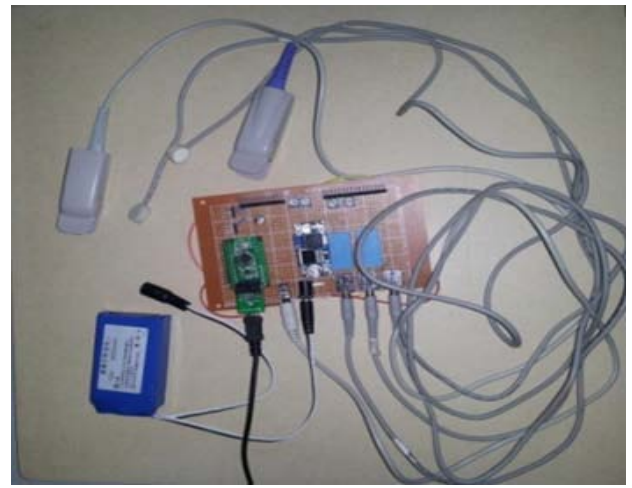

Fig. 7 collecting system of pulse sensors

As we can see there seems to be a small pulse present. But that is not nearly enough to get a proper readout. We needed to have a much stronger signal. Before we can amplify this signal we have to get rid of the DC part of the signals. We only want to know the pulse which is a relative signal in the output somewhere between $1 \mathrm{~Hz}$ and $20 \mathrm{~Hz}$. we needed a high pass filter to get the relative signal. This can be later amplified. Low pass filter was added to further smooth the signal. As you can see the reference point for the signal was VCC/3, about $1.7 \mathrm{~V}$ instead of GND. This made the signal oscillate around 1.7V. See Fig. 8.

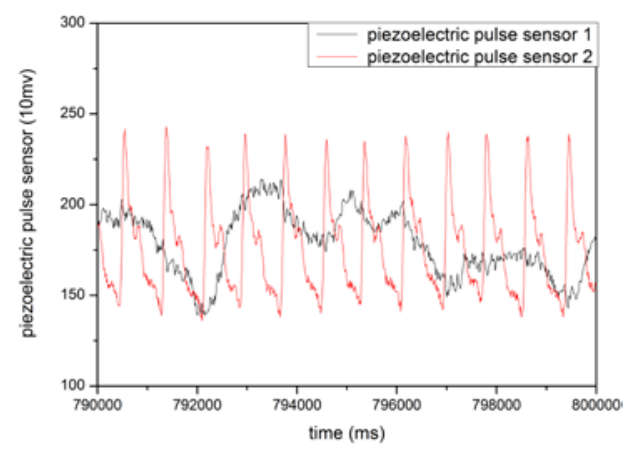

Fig. 8 time series of piezoelectric pulse sensor

There is still some noise in the signal ,most of people think that most of the imprecision and noise is to blame the fact that this whole experiment was done on no excellent PCB and signal cable, but we think this signal includes many information that we don't recognize so it is noise thought is inappropriate .

\section{IV . Obtaining heartbeat information with infrared pulse sensor}

Pulse oximetry is a non-invasive method allowing the monitoring of the saturation of the person's hemoglobin. The blood carries oxygen from the lungs to the rest of the body through arteries and the blood then returns through veins. when there is a good strong pulse in the finger, This change in color. Accordingly, the heartbeat information is detected, usually heartbeat information with infrared pulse sensor could be measured to use the tip of the finger (the thin part of the people's body) putting on the sensor, it was obvious that infrared light had to be used for illumination. It should be matched in wavelength. And with a flat finger sensor it was clear that a right angle illumination through the finger would be ideal. You can clearly see that we read out the absolute signal to detect if there is a finger on the sensor and the relative signal to detect the pulse.

As you can see we only get the relative signal and removed a good amount of the noise. The peaks are the artefacts of the steep signal changes when I put my finger on the sensor. And after the peaks you see some very small glimpse of the pulse. depicted in Fig. 9.

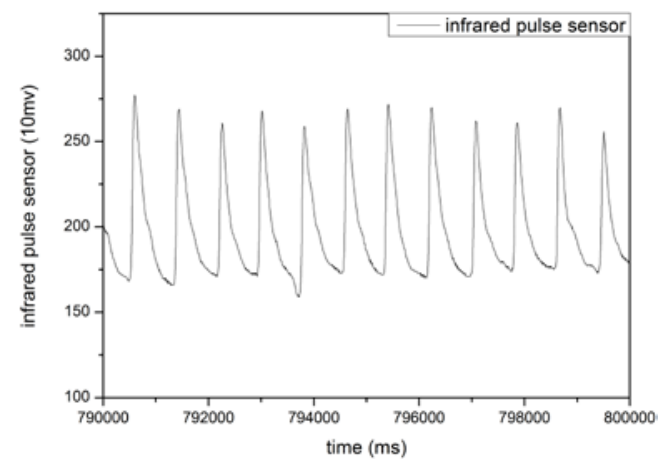

Fig. 9 time series of infrared pulse sensor 


\section{V . Obtaining heartbeat information with ECG}

ECG is used to measure the rate and regularity of heartbeats, it can present many damage of the heart, ECG is a interpretation of the electrical activity of the heart over a period of time and significant in long-term on line monitoring of cardiac activity for timely detection of abnormal heart conditions, The ECG device detects and amplifies the tiny electrical changes on the skin that are caused when the heart muscle depolarizes during each heartbeat.12-lead ECG is one in which 12 different electrical signals are recorded at approximately the same time and will often be used as a one-off recording of an ECG, ECG is the best way to measure and diagnose abnormal rhythms of the heart, and it can recognition emotion with the rhythms of the heartbeat, depicted in Fig. 10 and Fig. 11.

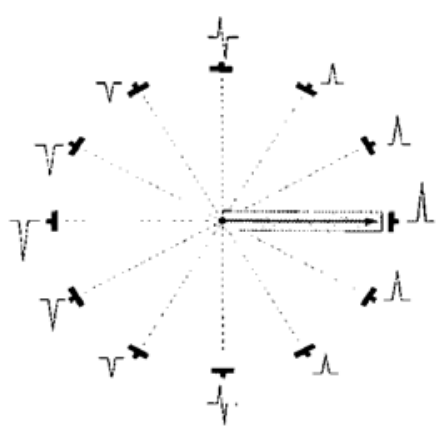

Fig. 10 ECG generate layout

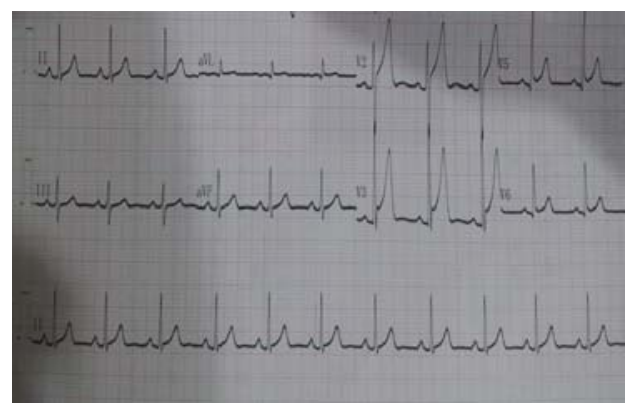

Fig. 11 Vectorcardiogram

\section{VI . Analysis}

At present, Medical care applications make use of medical and environmental sensors in order to obtain comprehensive health status information of the elderly and the patients, they will assume a more important role using very tiny sensors integrated into clothing or video cameras or small sensors in the external environment that are not carried by the patients and the elderly. Piezoelectric pulse sensor, infrared pulse sensor and ECG are wearable sensor devices with collecting body heartbeat dynamic information, this heavy and highly obtrusive sensory devices affect the freedom of movement of the people. Unobtrusiveness becomes a major challenge.

Obtaining heartbeat information with pressure cushion is good method, it can collect heartbeat information without impacting people action.

\section{Conclusion and Next work}

Heartbeat information collected through the above four ways, because the heartbeat information collected to use different physical pattern, we can see that many features is inconsistent, especially heartbeat information is acquired by piezoelectric pulse sensor, according to traditional Chinese medicine theory, it contains the human heart, organs, blood vessels, and even psychological and emotional information.

So, acquisition raw data of heartbeat is just on the battlefield "tickets", and extracting useful heartbeat information is fighting to win the "certificate". Next work are research in emotion recognition according to these system get data ,and exploit relation between the features information of emotional state and heartbeat information, improve emotion recognition accuracy.

\section{References}

[1] Eastwood, M. R. The Relation Between Physical and Mental Illness: The Physical Status of Psychiatric Patients at a Multiphasic Screening Survey. Clarke Institute of Psychiatry, 1975.

[2] Smolke, C. D.,Silver, P. A. Informing biological design by integration of systems and synthetic biology. Cell, 2011, 144 (6): 855-859.

[3] Purnick, P. E.,Weiss, R. The second wave of synthetic biology: from modules to systems. Nature Reviews Molecular Cell Biology, 2009, 10 (6): 410-422.

[4] Li, W.,Bao, J.,Shen, W. Collaborative wireless sensor networks: A survey. In 2011 IEEE International Conference on Systems, Man, and Cybernetics, SMC 2011, October 9, 2011 - October 12, 2011, Anchorage, AK, United states, 2011; 2614-2619.

[5] Parkka, J.,Ermes, M.,van Gils, M. Automatic feature selection and classification of physical and mental load using data from wearable sensors. In Information Technology and Applications in Biomedicine (ITAB), 2010 10th IEEE International Conference on, 2010; 1-5.

[6] Lakens, D. Using a Smartphone to Measure Heart Rate Changes During Relived Happiness and Anger. IEEE Transactions on Affective Computing, 2013, 1.

[7] Wu, H.-Y. Eulerian Video Processing and medical applications. Massachusetts Institute of Technology 2012.

[8] Causes of Irregular heartbeat. http://www.rightdiagnosis.com/symptoms/irregular_heartbeat/causes.htm

[9] Ohtaki, Y.,Papastefanou, G. A method of indoor ambulatory monitoring for psycho-physiological and behavioral activity assessment. In 6th China-Japan International Conference on Mechatronics, CJCM'2010, September 10, 2010 - September 12, 2010, Zhenjiang, China, 2011; 187-191.

[10] Lee, Y.-G.,Jeong, W. S.,Yoon, G. Smartphone-based mobile health monitoring. Telemedicine and e-Health, 2012, 18 (8): 585-590.

[11] Allen, J. Photoplethysmography and its application in clinical physiological measurement. Physiological measurement, 2007, 28 (3): R1-39.

[12] Wu, H.-Y.,Rubinstein, M.,Shih, E., etc. Eulerian video magnification for revealing subtle changes in the world. ACM Transactions on Graphics (TOG), 2012, 31 (4): 65-72.

[13] Bao, J.,Li, W.,Li, J., etc. Sitting Posture Recognition based on data fusion on pressure cushion. TELKOMNIKA Indonesian Journal of Electrical Engineering, 2013, 11 (4): 1769-1775. 